\begin{tabular}{|l|l|}
\hline $\begin{array}{l}\text { Instituto de } \\
\text { Geriatria e Gerontologia }\end{array}$ & $\begin{array}{l}\text { Pan American Journal of Aging Research } \\
\text { PAJAR, Porto Alegre, v. 8, p. 1-9, jan.-dez. } 2020 \\
\text { ISSN-L: 2357-9641 }\end{array}$ \\
\hline (6) ${ }_{\text {http://dx.doi.org/10.15448/2357-9641.2020.1.34072 }}$ &
\end{tabular}

ARTIGO ORIGINAL

\title{
Inadequação da ingestão alimentar em idosos: interação fármaco-nutriente
}

\author{
Inadequate food intake in elderly: drug-nutrient interaction \\ Ingesta inadecuada de alimentos en los ancianos: interacción entre medicamentos y \\ nutrientes
}

\section{Carina Duarte Venturini ${ }^{1}$ \\ orcid.org/0000-0002-8357-2041 \\ venturinicdv@gmail.com}

Paula Engroff ${ }^{2}$

orcid.org/0000-0002-3639-545X

paula.engroff@pucrs.br

Vanessa Sgnaolin ${ }^{2}$

orcid.org/0000-0002-9914-7146

vanessa.sgnaolin@pucrs.br

\section{Raquel Milani El Kik ${ }^{2}$}

orcid.org/0000-0001-6149-3089

raquel.elkik@pucrs.br

\section{Fernanda Bueno}

Morrone ${ }^{2}$

orcid.org/0000-0002-2709-2801

fernanda.morrone@pucrs.br

\section{Irenio Gomes 2}

orcid.org/0000-0003-2083-5059

irenio@nienpe.org

Recebido em: 3 mai. 2019. Aprovado em: 11 nov. 2019 Publicado em: 15 jun. 2020

(c) (i)

Artigo está licenciado sob forma de uma licença Creative Commons Atribuição 4.0 Internacional.

\section{Nutrição e uso de fármacos em idosos}

\section{Resumo}

Objetivos: avaliar a inadequação do consumo alimentar e a depleção estimadas de nutrientes causada pelo uso de fármacos em idosos.

Métodos: estudo populacional transversal, onde participaram 427 idosos. 0 consumo alimentar foi obtido a partir do Recordatório Alimentar de 24 horas e da Investigação da História Dietética. O uso de medicamentos foi avaliado por um questionário farmacoterapêutico.

Resultados: Dos idosos, 85,8\% utilizam medicamentos, sendo os mais prevalentes inibidores da enzima conversora de angiotensina (29,2\%), ácido acetilsalicilico $(28,7 \%)$, hidroclorotiazida $(24,4 \%)$, inibidores da enzima hidroximetilglutaril coenzima A redutase (17,0\%), carbonato de cálcio $(12,6 \%)$ e inibidores das bombas de prótons $(8,2 \%)$. Ácido acetilsalicilico, hidroclorotiazida e inibidores das bombas de prótons são os fármacos que possivelmente depletam o maior número de nutrientes. Dos usuários de inibidores da enzima hidroximetilglutaril coenzima A redutase, $97 \%$ poderiam possuir inadequação para vitamina $\mathrm{E}$; de diuréticos poupadores de potássio, 90,0\% inadequação para magnésio; de digoxina, 83.3\% inadequação para magnésio e 58,3\% para cálcio e potássio

Conclusões: os fármacos mais utilizados por idosos são responsáveis pela possivel depleção de inúmeros micronutrientes, muitas vezes associado a uma alta prevalência de inadequação de consumo, podendo acarretar possíveis deficiências nutricionais

Palavras-chave: desnutrição, envelhecimento, polimedicação, deficiências nutricionais.

\section{Abstract}

Aims: to evaluate the inadequacy of food consumption and the estimated nutrient depletion caused by the use of drugs in the elderly.

Methods: a cross-sectional population study, attended by 427 elderly. Food consumption was obtained from the 24-hour Food Recall Survey and the Research Dietetic History. The use of drugs was assessed by a pharmacotherapeutic questionnaire. Results: $85.8 \%$ of elderly use medications, the most prevalent inhibitors angiotensin converting enzyme inhibitor (29.2\%), acetylsalicylic acid (28.7\%), hydrochlorothiazide (24.4\%), hydroxymethyl-glutaryl coenzyme A reductase enzyme inhibitor (17.0\%), calcium carbonate (12.6\%) and proton pump inhibitors (8.2\%). Acetylsalicylic acid, hydrochlorothiazide and proton pump inhibitors are the drugs that possibly deplete the greatest number of nutrients. Users of hydroxymethyl-glutaryl coenzyme A reductase enzyme inhibitor, $97 \%$ could had inadequate for vitamin E; of potassium-sparing diuretics, $90.0 \%$ of inadequacy for magnesium; digoxin, $83.3 \%$ of inadequate for magnesium and 58.3\% for calcium and potassium.

Conclusions: the drugs most commonly used by elderly are responsible for the depletion of numerous micronutrients, often associated with a high prevalence of inadequate consumption, which may cause possible nutritional deficiencies. Keywords: malnutrition, aging, polypharmacy, deficiency diseases. 


\section{Resumen}

Objetivos: evaluar la inadecuación del consumo alimentario y la depleción estimada de nutrientes causada por el uso de fármacos en ancianos.

Métodos: estudio poblacional transversal, donde participaron 427 ancianos. El consumo alimentario fue obtenido a partir de la Encuesta Recordatoria de 24 Horas e Investigación de la Historia Dietética. El uso de medicamentos se evaluó mediante un cuestionario farmacoterapéutico.

Resultados: de los ancianos 85,8\% utilizan medicamentos, siendo los más prevalentes inhibidores de la enzima convertidora de angiotensina (29,2\%), ácido acetilsalicílico $(28,7 \%)$, hidroclorotiazida $(24,4 \%)$, inhibidores de la enzima hidroximetilglutaril coenzima La reductasa $(17,0 \%)$, carbonato de calcio $(12,6 \%)$ e inhibidores de las bombas de protones ( $8,2 \%$ ). El ácido acetilsalicilico, hidroclorotiazida e inhibidores de las bombas de protones son los fármacos que posiblemente deplete mayor número de nutrientes. De los usuarios de inhibidores de la enzima hidroximetilglutaril coenzima La reductasa, el 97\% tenía inadecuación para la vitamina E; de diuréticos ahorradores de potasio, 90,0\% inadecuación para el magnesio; de digoxina, 83,3\% inadecuación para magnesio y $58,3 \%$ para calcio y potasio.

Conclusiones: los fármacos más utilizados por ancianos son responsables de la depleción de innumerables micronutrientes, muchas veces asociado a una alta prevalencia de inadecuación de consumo, pudiendo acarrear posibles deficiencias nutricionales.

Palabras clave: desnutrición, envejecimiento, polifarmacia, enfermedades carenciales.

\section{Introdução}

O uso de fármacos pode acarretar importantes perdas de nutrientes, aumentando o risco de deficiências nutricionais e perda de peso em idosos ${ }^{1}$. Deve-se considerar que diversos medicamentos utilizados muitas vezes em associação, produzem efeitos adversos que incidem sobre o comportamento alimentar, levando à redução da ingestão de alimentos². Em idosos, a anorexia tem sido fortemente associada a diversas causas, tais como: redução do olfato e do paladar que por si só diminuem o prazer pela refeição, e exacerbada em individuos que utilizam vários medicamentos. Portanto, esse conjunto de fatores predispõe o idoso à desnutrição, à piora do quadro clínico e ao aumento da mortalidade?

Mesmo em casos de sobrepeso e de obesidade, a perda de peso em idosos eleva consideravelmente o risco de quedas, fraturas, institucionalização, fragilidade e morte ${ }^{4}$. Estudos epidemiológicos apontam que entre cinco e 10\% dos idosos em cuidados domiciliares, eaté $50 \%$ dos que recebem alta hospitalar, estão desnutridos ${ }^{5}$. A perda de peso, diminuição de massa magra e consequente aumento de gordura corporal leva a uma elevação de toxinas circulantes, em grande parte lipossolúveis e estocadas no tecido adiposo. Dessa forma, aumenta a toxicidade de fármacos lipossolúveis, provocando também um aumento de efeitos adversos ${ }^{6}$.

A maior expectativa de vida, principalmente em paises em desenvolvimento, elevou a prevalência de doenças crônicas e, também, o uso de múltiplos fármacos ${ }^{7.8}$. O idoso possui mais sucetibilidade à desnutrição, motivo pelo qual a presença de doença, aliada ao uso de fármacos, pode exacerbar a perda de nutrientes ${ }^{2.9}$. Dessa forma, a qualidade da alimentação diária é de suma importância a fim de atenuar, ou até mesmo evitar, grandes perdas nutricionais provocadas pela associação de fatores, como: idade, doença e uso de fármacos. Portanto, o objetivo deste estudo foi avaliar a inadequação do consumo alimentar e a depleção estimadas de nutrientes causada pelo uso de fármacos em idosos.

\section{Métodos}

Entre 2005 e 2006 foi realizado um estudo transversal pelo Instituto de Geriatria e Gerontologia da Pontificia Universidade Católica do Rio Grande do Sul, em parceria com a Prefeitura Municipal de Porto Alegre, Brasil, denominado: Estudo Multidimensional dos Idosos de Porto Alegre. Nesse estudo participaram 512 idosos com idade igual ou maior que 60 anos, representando uma amostra aleatória dos residentes do município. O cálculo amostral foi baseado no número estimado de individuos para cada bairro de Porto Alegre, conforme o censo populacional do ano $2000^{10}$.

O estudo gerou um banco de dados com diversos indicadores epidemiológicos do qual, para o presente estudo, foram utilizados o perfil farmacoterapêutico e a ingestão de nutrientes. A coleta de dados foi realizada por profissionais das Faculdades de Farmácia e Nutrição da Pontificia Universidade Católica do Rio Grande do Sul, com o auxilio de alunos devidamente treinados para a aplicação 
dos instrumentos de pesquisa. Dos 512 idosos que inicialmente participaram do estudo, 85 foram excluidos do banco de dados por apresentarem informações incorretas e/ou incompletas, resultando em uma amostra final de 427 idosos.

O uso de medicamentos foi avaliado a partir de uma entrevista conduzida por acadêmicos de farmácia, que utilizaram um questionário farmacoterapêutico. Os idosos relatavam com base na memória e em elementos de suporte, tais como embalagens, prescrições e bulas, as informações sobre regimes terapêuticos, medicamentos prescritos e utilizados, frequência de uso e automedicação.

Foram selecionados os fármacos ou classes farmacológicas mais utilizadas e responsáveis pela perda de nutrientes ${ }^{11,12}$, tais como: ácido acetilsalicilico, digoxina, verapamil, furosemida, hidroclorotiazida, metformina, carbonato de cálcio, diurético poupador de potássio, anti-hipertensivo inibidor da enzima conversora de angiotensina, antiulceroso antagonista dos receptores de histamina $\mathrm{H} 2$, antiulceroso inibidor da bomba de prótons, hipolipemiante inibidor da enzima hidroximetil-glutaril coenzima A redutase, antidepressivo inibidor seletivo da recaptação de serotonina.

A análise da ingestão de nutrientes foi feita a partir da coleta de dados de consumo alimentar. Para isso, foram utilizados o Recordatório Alimentar de 24 horas e a Investigação da História Dietética. A composição nutricional da dieta foi calculada com o auxílio do programa NutWin versão 2.5. Os resultados foram expressos em microgramas para as vitaminas A, B9 e B12, e em miligramas para os demais nutrientes. Foi avaliado o consumo de vitaminas (A, C, E, B1, B2, B3, B6, ácido fólico e B12) e minerais: cálcio, magnésio, ferro, sódio, potássio, zinco e fósforo. Para verificar a ingestão de nutrientes, a quantidade consumida foi comparada com os valores de referência estabelecidos pelo The Institute of Medicine (2006) e Ross et al. (2011) para o mineral cálcio, de acordo com sexo e idade ${ }^{13,14}$.

Foi considerado inadequado o consumo abaixo do Requerimento Médio Estimado (EAR, do inglês Estimated Average Requirement) e acima do Nivel Máximo Tolerado (UL, do inglês Tolerable Upper Intake Level). Devido ao fato de que nem todos os nutrientes possuem recomendações estabelecidas de EAR e UL, foi considerado inadequado o consumo acima da UL para os nutrientes que não possuem EAR e abaixo da EAR para os que não possuem UL.

Através da revisão da literatura, foram conhecidos os nutrientes possivelmente depletados pelos fármacos ou classe farmacológica e avaliada a prevalência de indivíduos com inadequação de ingestão de cada nutriente. Tais resultados possibilitam inferir sobre o déficit de micronutrientes devido a uma ingestão inadequada e exacerbada pelo uso de medicamentos.

Foi utilizado o programa estatístico Statistical Package for Social Sciences (SPSS) versão 17.0 para análise e tabulação dos dados. A frequência de uso de medicamentos foi descrita em número total e porcentagem. Esse estudo foi aprovado pelo Comitê de Ética em Pesquisa da Pontifícia Universidade Católica do Rio Grande do Sul sob parecer número 0502935. Todos os participantes assinaram o Termo de Consentimento Livre e Esclarecido.

\section{Resultados}

Participaram do estudo 427 individuos, dos quais a maioria (85,8\%) fazia uso de medicamentos. Os dados sociodemográficos e que descrevem detalhadamente essa população foram publicados por Venturini et al. ${ }^{15}$

A Tabela 1 apresenta os resultados do estudo, ou seja, os fármacos em ordem decrescente de frequência de uso e a possivel prevalência de inadequação da ingestão de nutrientes, bem como dados provenientes da revisão da literatura, os nutrientes que podem sofrer depleção ou aumento pela ação de fármaco e os efeitos adversos relacionados ao comprometimento do equilibrio nutricional. Dentre os fármacos analisados, os de ação cardiovascular foram os mais utilizados, sendo os de uso mais frequente o anti-hipertensivo inibidor da enzima conversora de angiotensina (29,4\%), o ácido acetilsalicilico $(29,1 \%)$ e o diurético tiazídico $(24,4 \%)$. 
TABELA 1 - Frequência de utilização de medicamentos e inadequação da ingestão de nutrientes em idosos $(N=427)$.

\begin{tabular}{|c|c|c|c|c|}
\hline $\begin{array}{l}\text { FÁRMACO / } \\
\text { CLASSIFICAÇÃO }\end{array}$ & $\begin{array}{l}\text { FREQUÊNCIA } \\
\text { DE USO N (\%) }\end{array}$ & $\begin{array}{l}\text { NUTRIENTES } \\
\text { DEPLETADOS } \\
\text { OU } \\
\text { AUMENTADOS* }\end{array}$ & $\begin{array}{l}\text { PREVALÊNCIA DE } \\
\text { INADEQUAÇÃO } \\
\text { DA INGESTÃO (\%) }\end{array}$ & $\begin{array}{l}\text { EFEITO ADVERSO } \\
\text { RELACIONADO AO } \\
\text { COMPROMETIMENTO } \\
\text { DO EQUILIBBRIO } \\
\text { NUTRICIONAL* }\end{array}$ \\
\hline $\begin{array}{l}\text { Anti-hipertensivo } \\
\text { inibidor da enzima } \\
\text { conversora da } \\
\text { angiotensina }\end{array}$ & $\begin{array}{l}109(29,4) \\
114(29,2) \\
114(29,2)\end{array}$ & $\begin{array}{c}\mathrm{Na} \\
\mathrm{Zn} \\
\mathrm{K}\end{array}$ & $\begin{array}{l}37,6 \\
43,0 \\
81,6\end{array}$ & $\begin{array}{l}\text { Tosse seca, hipotensão, } \\
\text { tontura, alteração do } \\
\text { paladar, náusea, vômito } \\
\text { diarréia, perda de } \\
\text { apetite, dor abdominal. }\end{array}$ \\
\hline $\begin{array}{l}\text { Ácido acetil } \\
\text { salicilico }\end{array}$ & $\begin{array}{l}104(29,1) \\
109(28,5) \\
111(28,6) \\
112(28,7) \\
107(28,8) \\
112(28,7)\end{array}$ & $\begin{array}{c}\text { ácido fólico } \\
\text { vitamina C } \\
\mathrm{Fe} \\
\mathrm{K} \\
\mathrm{Na} \\
\mathrm{Zn}\end{array}$ & $\begin{array}{c}63,5 \\
42,2 \\
5,4 \\
82,1 \\
42,1 \\
45,5\end{array}$ & $\begin{array}{c}\text { Anemia, insônia, dor } \\
\text { de estômago, gastrite, } \\
\text { úlcera, náuseas, vômito, } \\
\text { perda de apetite. }\end{array}$ \\
\hline $\begin{array}{l}\text { Diurético tiazídico } \\
\text { (Hidroclorotiazida) }\end{array}$ & $\begin{array}{l}95(24,4) \\
94(24,4) \\
95(24,4) \\
90(24,2) \\
90(24,3) \\
94(24,2)\end{array}$ & $\begin{array}{c}\mathrm{K} \\
\mathrm{Mg} \\
\mathrm{Zn} \\
\mathrm{P} \\
\mathrm{Na} \\
\mathrm{Ca}\end{array}$ & $\begin{array}{r}83,2 \\
79,8 \\
41,1 \\
14,4 \\
43,3 \\
74,5\end{array}$ & $\begin{array}{c}\text { Fraqueza, fadiga, } \\
\text { aumento do colesterol } \\
\text { sérico. }\end{array}$ \\
\hline $\begin{array}{l}\text { Inibidor da } \\
\text { hidroximetilglutaril } \\
\text { coenzima A } \\
\text { redutase (Estatinas) }\end{array}$ & $66(17,0)$ & vitamina $\mathrm{E}$ & 97,0 & $\begin{array}{c}\text { Cefaleia, dor abdominal } \\
\text { flatulência, constipação, } \\
\text { náusea, diarreia, dor } \\
\text { muscular e insônia. }\end{array}$ \\
\hline $\begin{array}{l}\text { Antiácido } \\
\text { carbonato de } \\
\text { cálcio }\end{array}$ & $\begin{array}{l}50(12,9) \\
46(12,4)\end{array}$ & $\begin{array}{c}\mathrm{Fe} \\
\mathrm{P}\end{array}$ & $\begin{array}{l}2,0 \\
6,5\end{array}$ & $\begin{array}{l}\text { Cefaleia, constipação, } \\
\text { náusea, vômito, dor } \\
\text { abdominal, perda de } \\
\text { apetite. }\end{array}$ \\
\hline $\begin{array}{l}\text { Antiulceroso } \\
\text { Inibidor da bomba } \\
\text { de prótons }\end{array}$ & $\begin{array}{l}32(8,2) \\
32(8,2) \\
30(8,1) \\
31(8,0) \\
28(7,8) \\
32(8,2)\end{array}$ & $\begin{array}{c}\mathrm{Fe} \\
\mathrm{Zn} \\
\mathrm{Na} \\
\text { vitamina B1 } \\
\text { ácido fólico } \\
\text { vitamina B12 }\end{array}$ & $\begin{array}{c}9,4 \\
53,1 \\
33,3 \\
29,0 \\
75,0 \\
21,9\end{array}$ & $\begin{array}{l}\text { Cefaléia, náusea, } \\
\text { vômito, dor abdominal, } \\
\text { flatulência, diarreia, } \\
\text { constipação. }\end{array}$ \\
\hline $\begin{array}{l}\text { Hipoglicemiante } \\
\text { (Metformina) }\end{array}$ & $\begin{array}{l}27(7.5) \\
29(7.5)\end{array}$ & $\begin{array}{l}\text { ácido fólico } \\
\text { vitamina B12 }\end{array}$ & $\begin{array}{l}63,0 \\
24,1\end{array}$ & $\begin{array}{l}\text { Náusea, vômito, } \\
\text { indigestão, flatulência, } \\
\text { diarreia, gosto metálico, } \\
\text { fraqueza, perda de } \\
\text { apetite. }\end{array}$ \\
\hline
\end{tabular}




\begin{tabular}{|c|c|c|c|c|}
\hline $\begin{array}{l}\text { FÁRMACO / } \\
\text { CLASSIFICAÇÃO }\end{array}$ & $\begin{array}{l}\text { FREQUÊNCIA } \\
\text { DE USO N (\%) }\end{array}$ & $\begin{array}{l}\text { NUTRIENTES } \\
\text { DEPLETADOS } \\
\text { OU } \\
\text { AUMENTADOS* }\end{array}$ & $\begin{array}{l}\text { PREVALÊNCIA DE } \\
\text { INADEQUAÇÃO } \\
\text { DA INGESTÃO (\%) }\end{array}$ & $\begin{array}{l}\text { EFEITO ADVERSO } \\
\text { RELACIONADO AO } \\
\text { COMPROMETIMENTO } \\
\text { DO EQUILÍBRIO } \\
\text { NUTRICIONAL* }\end{array}$ \\
\hline $\begin{array}{l}\text { Antidepressivo } \\
\text { Inibidor seletivo } \\
\text { da recaptação de } \\
\text { serotonina }\end{array}$ & $28(7,2)$ & $\mathrm{Fe}$ & 7,1 & $\begin{array}{c}\text { Xerostomia, constipação, } \\
\text { insônia, cefaleia, náusea, } \\
\text { nervosismo, ansiedade, } \\
\text { fraqueza muscular e } \\
\text { tremores. }\end{array}$ \\
\hline $\begin{array}{l}\text { Bloqueador de } \\
\text { canais de cálcio } \\
\text { (Verapamil) }\end{array}$ & $\begin{array}{l}26(6.7) \\
26(6.7)\end{array}$ & $\begin{array}{l}\mathrm{Ca} \\
\mathrm{Zn}\end{array}$ & $\begin{array}{l}65,4 \\
46,2\end{array}$ & $\begin{array}{c}\text { Constipação intestinal, } \\
\text { edema, cefaleia, } \\
\text { náuseas, tonturas, } \\
\text { dispneia e fadiga. }\end{array}$ \\
\hline $\begin{array}{l}\text { Diurético de Alça } \\
\text { (Furosemida) }\end{array}$ & $\begin{array}{l}25(6,4) \\
25(6,4) \\
23(6,2) \\
25(6,5) \\
25(6,4) \\
24(6,5)\end{array}$ & $\begin{array}{l}\mathrm{Ca} \\
\mathrm{K} \\
\mathrm{Na} \\
\mathrm{Mg} \\
\mathrm{Zn} \\
\mathrm{P}\end{array}$ & $\begin{array}{c}72,0 \\
80,0 \\
47,8 \\
76 \\
36,0 \\
8,3\end{array}$ & $\begin{array}{c}\text { Tontura, febre, } \\
\text { inquietação, desconforto } \\
\text { gástrico, câimbras, } \\
\text { náusea, vômito, perda de } \\
\text { apetite. }\end{array}$ \\
\hline $\begin{array}{l}\text { Antiulceroso } \\
\text { antagonista } \mathrm{H}_{2}\end{array}$ & $\begin{array}{l}14(3,6) \\
14(3,6) \\
14(3,6) \\
14(3,6) \\
14(3,6) \\
12(3,4) \\
14(3,6)\end{array}$ & $\begin{array}{c}\mathrm{Ca} \\
\mathrm{Zn} \\
\mathrm{Mg} \\
\mathrm{Fe} \\
\text { vitamina B1 } \\
\text { ácido fólico } \\
\text { vitamina B12 }\end{array}$ & $\begin{array}{c}78,6 \\
64,3 \\
78,6 \\
7,1 \\
57,1 \\
83,3 \\
7,1\end{array}$ & $\begin{array}{c}\text { Tontura, sonolência, } \\
\text { cefaleia, náusea, vômito, } \\
\text { diarreia. }\end{array}$ \\
\hline Digoxina & $\begin{array}{l}12(3,1) \\
12(3,1) \\
11(3,0) \\
12(3,1) \\
12(3,1)\end{array}$ & $\begin{array}{c}\mathrm{Mg} \\
\mathrm{Ca} \\
\mathrm{P} \\
\mathrm{K} \\
\text { vitamina } \mathrm{B} 1\end{array}$ & $\begin{array}{c}83,3 \\
58,3 \\
9,1 \\
58,3 \\
16,7\end{array}$ & $\begin{array}{l}\text { Náusea, vômito, dor } \\
\text { abdominal, diarreia, } \\
\text { tontura, cefaleia, } \\
\text { fraqueza. }\end{array}$ \\
\hline $\begin{array}{l}\text { Diurético poupador } \\
\text { de potássio }\end{array}$ & $\begin{array}{l}10(2,6) \\
10(2,6) \\
10(2,7) \\
10(2,7) \\
10(2,8) \\
10(2,6)\end{array}$ & $\begin{array}{c}\mathrm{Ca} \\
\mathrm{Mg} \\
\mathrm{Na} \\
\mathrm{P} \\
\text { ácido fólico } \\
\mathrm{K}\end{array}$ & $\begin{array}{c}70,0 \\
90,0 \\
30,0 \\
0 \\
70,0 \\
90,0\end{array}$ & $\begin{array}{l}\text { Sonolência, desconforto } \\
\text { gástrico, náusea, vômito. }\end{array}$ \\
\hline
\end{tabular}

*Fonte: Vagnini \& Fox, 2005; Martins et al., 2003. (): aumento. Minerais: cálcio (Ca), magnésio (Mg), ferro (Fe), sódio $(\mathrm{Na})$, potássio $(\mathrm{K})$, zinco $(\mathrm{Zn})$, fósforo $(\mathrm{P})$

O ácido acetilsalicilico, a hidroclorotiazida e os inibidores das bombas de prótons foram os que possivelmente depletam maior número de nutrientes, especialmente, os minerais como ferro, zinco, sódio e potássio, cuja perda é acentuada mediante a associação de medicamentos. Embora com menor frequência de uso, a digoxina, os diuréticos (furosemida e poupadores de potássio) 
e os antiulcerosos, antagonista $\mathrm{H} 2$, foram responsáveis pelas prováveis perdas de diversos minerais, principalmente cálcio e magnésio, cuja perda é comum a todos.

Os resultados apontam que o uso de fármacos pode causar maior prejuizo de minerais do que vitaminas, embora algumas classes podem aumentar os niveis séricos de minerais, como os diuréticos poupadores de potássio e os antihipertensivos inibidores da enzima conversora de angiotensina, ambos relacionados com retenção de potássio e, a hidroclorotiazida, de cálcio. Em relação às vitaminas, as do complexo B (B1, B12 e ácido fólico) figuram com maior frequência na lista dos micronutrientes que podem sofrer depleção por fármacos, sendo eles: ácido acetilsalicilico, digoxina, diuréticos poupadores de potássio, metformina, antiulcerosos antagonista $\mathrm{H} 2 \mathrm{e}$ inibidores das bombas de prótons.

Em relação à inadequação da ingestão de nutrientes, nossos resultados sugerem que os inibidores da enzima hidroxi-metil-glutaril coenzima A redutase podem depletar vitamina E, sendo que $97,0 \%$ dos usuários podem possuir inadequação de consumo desse nutriente. Em seguida, os diuréticos poupadores de potássio podem depletar magnésio, sendo que $90,0 \%$ dos seus usuários podem possuir inadequação da ingestão desse mineral. A digoxina pode estar envolvida com a perda de magnésio, cálcio e potássio, cuja frequência de inadequação da ingestão desses nutrientes foi de 83.3\% para o magnésio e 58,3\% para cálcio e potássio. Entre os usuários de antiácidos antagonista $\mathrm{H} 2$, que estão relacionados com a depleção de ácido fólico, $83.3 \%$ desses podem possuir inadequação da ingestão dessa vitamina. Alguns fármacos são possiveis depletores de potássio, cujos usuários possuem alta prevalência de inadequação da ingestão desse nutriente: hidroclorotiazida (83,2\%), ácido acetilsalicílico $(82,1 \%)$ e furosemida $(80,0 \%)$.

\section{Discussão}

O uso de fármacos, isolados ou em associação, pode levar a deficiências nutricionais por diferentes vias como: aumento da excreção, depleção das reservas, redução da absorção, aumento da necessidade e alteração da atividade de um ou mais nutrientes ${ }^{11}$. O presente estudo analisou os principais fármacos utilizados por idosos e os respectivos nutrientes possivelmente depletados, relacionando-os com a inadequação da ingestão desses mesmos nutrientes. Nesse contexto, a análise de inadequação mostrou o percentural de individuos, cuja injestão de nutrientes encontrase abaixo da recomendação mínima diária ${ }^{13}$. Nossos resultados sugerem que os fármacos mais utilizados por idosos são responsáveis pela perda de minerais como sódio, potássio, zinco, ferro, fósforo, cálcio e magnésio, além das vitaminas C, E, B1, B12 e ácido fólico. A maioria desses fármacos produz efeitos adversos que afetam o trato disgestório, conduzindo o idoso a uma redução da ingestão de alimentos. Esses achados corroboram com um estudo recente realizado com idosos não institucionalizados, demonstrando que o uso diário de 3 ou mais fármacos está associado com baixos niveis séricos de vitaminas D, K, B6 e folato ${ }^{16}$.

Éalta a prevalência de inadequação da ingestão de nutrientes entre os usuários de medicamentos, o que aumenta o risco de deficiências nutricionais comuns nessa faixa etária ${ }^{1}$. De forma geral, a deficiência de nutrientes pode ocasionar desequilibrio de eletrólitos, desordens cardiovasculares, espasmos musculares, comprometimento da resposta imunológica, anemias, transtornos de humor, entre outros ${ }^{17.18}$. A deficiência de nutrientes pode exacerbar os efeitos adversos causados pelos fármacos, como por exemplo: fraqueza, fadiga, tontura, hipotensão, constipação, cãimbras, insônia e perda de apetite ${ }^{11.19}$, induzindo o idoso à busca por tratamentos farmacológicos adicionais, como a automedicação. Dessa forma, aumenta o uso de laxantes, antiácidos, anti-inflamatórios e ansiolíticos que podem elevam ainda mais a perda de nutrientes e o aparecimento de efeitos adversos, inclusive com o desenvolvimento de novas patologias ${ }^{20,21}$.

Além dos nutrientes citados nesse estudo. alguns outros compostos de vital importância ao organismo também são perdidos pelo uso de medicamentos. É o caso da ubiquinona 
ou coenzima Q10, glutationa, manganês e betacaroteno. O uso de diuréticos tiazídicos, anti-hipertensivos betabloqueadores e estatinas, entre outros fármacos, podem reduzir significativamente os estoques de coenzima Q10 ${ }^{11}$. Envolvida diretamente na produção de energia, é sintetizada em tecidos ricos em mitocôndria, como o coração por exemplo, cuja deficiência é responsável pela sensação de cansaço e falência cardiaca. Possui atividade antioxidante, prevenindo inclusive a oxidação do colesterol22.23. No entanto, pode ser depletada justamente por fármacos largamente utilizados nos tratamentos de cardiopatias e dislipidemias ${ }^{24}$. Deve-se ressaltar a ocorrência de possiveis perdas de coenzima Q10 em tratamentos combinados, em que todos os fármacos utilizados são responsáveis pela sua depleção, resultando em efeitos adversos como fraqueza, fadiga e dores musculares. Tal situação foi encontrada repetidas vezes no presente estudo. As doenças cardiovasculares possuem caráter inflamatório e, portanto, oxidativo ${ }^{25}$. Já o uso de estatinas, também depleta vitamina $\mathrm{E}^{26}$, importante agente antioxidante, cuja frequência de inadequação da ingestão foi de quase 100\% entre os usuários dessa classe de fármacos.

A glutationa é o principal antioxidante do organismo, cujo estoque é severamente diminuido devido à sua utilização no metabolismo hepático do paracetamol e do ácido acetilsalicilico ${ }^{27}$. Entre outras funções, a glutationa é responsável por manter as vitaminas $\mathrm{C}$ e $\mathrm{E}$ em suas formas ativas $^{28}$. O ácido acetilsalicilico, que por sua vez depleta vitamina C, é usado de forma crônica em algumas cardiopatias, motivo pelo qual causa lesão da mucosa gástrica, além de depletar vários nutrientes, como por exemplo, ácido fólico, ferro, sódio e zinco ${ }^{12.29}$. Muitos deles também são perdidos devido ao uso crônico e concomitante de omeprazol, um antiulceroso inibidor das bombas de prótons, altamente recomendado para reduzir o desconforto gástrico causado pelo ácido acetilsalicilico ${ }^{30}$. Ambos os medicamentos causam efeitos adversos que podem resultar na redução da ingestão de alimentos. Tais efeitos incluem náuseas, vômitos e desconforto gástrico, resultando em uma associação propícia ao desenvolvimento de anemia microcitica, devido à deficiência de ferro, e megaloblástica, causada pela deficiência de ácido fólico e vitamina B12 ${ }^{16,19,31}$. Inibidores da bomba de prótons diminuem a formação de ácido clorídrico, impedindo assim a formação de fator intrínseco, dependente de $\mathrm{pH}$ ácido e necessário à absorção de vitamina B12 pelo íleo ${ }^{32}$. Embora esse estudo tenha mostrado baixa prevalência de uso de omeprazol, alguns autores revelam que é frequente o uso desse medicamento, inclusive como primeira escolha no tratamento de gastrite e úlcera em idosos ${ }^{31}$.

Curiosamente, nossos resultados demonstram que os fármacos que provavelmente elevam os niveis séricos de alguns minerais, como potássio e cálcio, apresentam alta prevalência de indivíduos com inadequação de consumo desses nutrientes. É o caso dos diuréticos poupadores de potássio, diuréticos tiazidicos e anti-hipertensivos inibidores da enzima conversora de angiotensina, todos com inadequação da ingestão acima de $80 \%$. Os dados sugerem que esse efeito de retenção de minerais, já conhecido entre os médicos, é previamente alertado ao paciente sobre a necessidade de reduzir a ingestão de cálcio e potássio, sob risco de comprometimento da função renal e cardiaca ${ }^{19,33}$. Contudo, esses mesmos fármacos podem depletar outros nutrientes, como magnésio e ácido fólico, ambos com alta prevalência de individuos com inadequação da ingestão. Isso pode ocorrer devido à baixa ingestão profilática de frutas e vegetais fontes de potássio magnésio e ácido fólico.

Devemos considerar algumas limitações do estudo. As informações sobre os medicamentos relatados pelos idosos foram baseadas não somente em prescrições, bulas e embalagens, mas também na memória dos entrevistados. Portanto, um viés de subnotificação pode ter ocorrido, além de confusão de nomes de medicamentos. Da mesma forma, alimentos e bebidas consumidos pelos idosos podem ter sofrido subnotificação. Além disso, um único dia de Recordatório Alimentar de 24 horas foi aplicado aos idosos, motivo pelo qual esse instrumento foi associado 
à história dietética. São necessários mais dias de inquéritos alimentares para assegurar precisão sobre o consumo e a inadequação da ingestão de nutrientes. A depleção de nutrientes provocada pelos fármacos utilizados foi estimada somente através de levantamento bibliográfico, devido à impossibilidade de análises bioquimicas por tratarse de um estudo retrospectivo realizado em banco de dados. Portanto, os dados aqui apresentados devem ser interpretados com cautela.

Os fármacos mais utilizados são responsáveis pela possivel depleção de micronutrientes, muitos deles apresentando também alta prevalência de inadequação da ingestão dos mesmos. O uso de medicamentos no idoso deve ser restrito ao necessário e acompanhado de uma adequada reposição de nutrientes, principalmente, em caso de politerapia medicamentosa.

\section{Referências}

1. Jyrkkä J, Mursu J, Enlund H, et al. Polypharmacy and nutritional status in elderly people. Curr Opin Clin Nutr Metab Care. 2012:15(1):1-6. https://doi. org/10.1097/mco.0b013e32834d155a

2. Jyrkkä J, Enlund H, Lavikainen P, et al. Association of polypharmacy with nutritional status, functional ability and cognitive capacity over a three-year period in an elderly population. Pharmacoepidemiol Drug Saf. 2011:20(5):514-22. https://doi.org/10.1002/pds.2116

3. Morley, John E. Decreased food intake with aging. J Gerontol A Biol Sci Med Sci. 2001:56(2):81-8.

4. Bales CW. Buhr G. Is obesity bad for older persons? A systematic review of the pros and cons of weight reduction in later life. J Am Med Dir Assoc. 2008:9(5):302-12. https://doi.org/10.1016/j.jamda.2008.01.006

5. Cansado P, Ravasco P, Camilo M. A longitudinal study of hospital undernutrition in the elderly: comparison of four validated methods. J Nutr Health Aging. 2009:13(2):159-64. https://doi.org/10.1007/ s12603-009-0024-y

6. Kim MJ, Marchand P, Henegar C, et al. Fate and complex pathogenic effects of dioxins and polychlorinated biphenyls in obese subjects before and after drastic weight loss. Environ Health Perspect. 2011;119(3):377-83. https://doi.org/10.1289/ehp.1002848

7. World Health Organization - WHO. Trends in life expectancy [Internet]. Genebra: WHO; 2012 [Capturado em 2012 Ago 01]. Disponivel em: http://www.who. int/whr/1998/media_centre/press_release/en/ index3.html.
8. Glick M. The health of the nation: why you should care. J Am Dent Assoc. 2007:138(2):144-6.

9. Morley JE. Undernutrition in older adults. Fam Pract. 2012;29:189-ig3.

10. Instituto Brasileiro de Geografia e Estatística IBGE. Resultados preliminares do censo 2010 [Internet]. Brasilia: IBGE; 2010 [Capturado em 2012 jun 10]. Disponivel em: http://www.ibge.gov.br. https://doi. org/10.5752/p.2175-5841.2012v10n28p1122

11. Vagnini F, Fox B. The side effects bible: the dietary solution to unwanted side effectos of common medications. New York: Broadway Books; 2005.

12. Gomez R, Venturini C. Interação entre alimentos e medicamentos. Porto Alegre: Suliani Letra e Vida Editora; 2009.

13. Institute of Medicine of The National Academies. Dietary reference intakes: the essential guide to nutrient requirements, 2006. [Capturado em $2012 \mathrm{apr}$ 22]. Disponivel em: http://www.nap.edu/openbook. php?record_id=11537\&page=R3.

14. Ross AC, Manson JE, Abrams SA, et al. The 2011 dietary reference intakes for calcium and vitamin D: what dietetics practitioners need to know. J Am Diet Assoc, 2011;111(4):524-7.

15. Venturini CD, Engroff $P$, Sgnaolin V, et al. Consumo de nutrientes em idosos residentes em Porto Alegre (RS), Brasil: um estudo de base populacional. Ciênc saúde coletiva. 2015; 20(12):3701-11. https://doi. org/10.1590/1413-812320152012.01432015

16. Fabian E, Bogner M, Kickinger A, et al. Intake of medication and vitamin status in the elderly. Ann Nutr Metab. 2011;58(2):118-25. https://doi. org/10.1159/000327351

17. Herrmann WJ, Lorenzl S, Obeid R. Review of the role of hyperhomocysteinemia and B-vitamin deficiency in neurological and psychiatric disorders--current evidence and preliminary recommendations. Fortschr Neurol Psychiatr. 2007;75(9):515-27.

18. Barbagallo M, Belvedere M, Dominguez LJ. Magnesium homeostasis and aging. Magnes Res. 2009:22(4):235-46.

19. Martins C, Moreira SM, Pierosan SR. Interações droga-nutriente. Curitiba: Nutroclínica; 2003.

20. Andreazza RS, Silveira De Castro M, et al. Causes of drug-related problems in the emergency room of a hospital in southern Brazil. Gac Sanit. 2011;25(6):5016. https://doi.org/10.1016/j.gaceta.2011.05.016

21. Ventura MT, Laddaga R, Cavallera P, et al. Adverse drug reactions as the cause of emergency department admission: focus on the elderly. Immunopharmacol Immunotoxicol. 2010;32(3):426-9. https://doi.org/10.3109/08923970903468847

22. Ren J, Pulakat L, Whaley-Connell A, et al. Mitochondrial biogenesis in the metabolic syndrome and cardiovascular disease. J Mol Med (Berl). 2010;88(10):993-1001. https://doi.org/10.1007/ s00109-010-0663-9 
23. Lee JH, Jarreau T, Prasad A, et al. Nutritional assessment in heart failure patients. Congest Heart Fail. 2011;17(4):199-203. https://doi.org/10.1111/j. 1751-7133.2011.00239.x

24. Deichmann R, Lavie C, Andrews S. Coenzyme q10 and statin-induced mitochondrial dysfunction. Ochsner J. 2010;10(1):16-21.

25. Nakbi A, Koubaa N, Ben Hamda K, et al. Association between oxidative stress parameters and inflammation markers according to the gravity of the acute coronary syndrome. Tunis Med. 2011;89(7):621-6.

26. Palomäki A, Malminiemi K, Malminiemi O, et al. Effects of lovastatin therapy on susceptibility of LDL to oxidation during alpha-tocopherol supplementation. Arterioscler Thromb Vasc Biol. 1999;19(6):1541-8. https://doi.org/10.1161/01.atv.19.6.1541

27. Masubuchi Y, Nakayama J, Sadakata Y. Protective effects of exogenous glutathione and related thiol compounds against drug-induced liver injury. Biol Pharm Bull. 2011:34(3):366-70. https://doi. org/10.1248/bpb.34.366

28. Clavel JP, Emerit J, Thuillier A. Lipid peroxidation and free radicals. Role in cellular biology and pathology. Pathol Biol (Paris). 1985:33(1):61-9.

29. Schulz HU, Schürer M, Krupp S, et al. Effects of acetylsalicylic acid on ascorbic acid concentrations in plasma, gastric mucosa, gastric juice and urine -- a double-blind study in healthy subjects. Int J Clin Pharmacol Ther. 2004;42(9):481-487. https://doi. org/10.5414/cpp42481

30. Sugimoto M, Nishino M, Kodaira C, et al. Esophageal mucosal injury with low-dose aspirin and its prevention by rabeprazole. J Clin Pharmacol. 2010;50(3):320-30. https://doi.org/10.1016/s00165085(09)62058-8

31. Greenwald DA. Aging, the gastrointestinal tract, and risk of acid-related disease. Am J Med. 2004;117:8S-13S

32. Kapadia C. Cobalamin (Vitamin B12) deficiency: is it a problem for our aging population and is the problem compounded by drugs that inhibit gastric acid secretion? J Clin Gastroenterol. 2000;30(1):4-6. https://doi.org/10.1097/00004836-200001000-00003

33. Coe FL, Parks JH, Bushinsky DA, et al. Chlorthalidone promotes mineral retention in patients with idiopathic hypercalciuria. Kidney Int. 1988;33(6):11406. https://doi.org/10.1038/ki.1988.122

\section{Carina Duarte Venturini}

Nutricionista. Doutora em Gerontologia Biomédica. Hospital de Aeronáutica de Canoas.

\section{Paula Engroff}

Farmacêutica. Doutora em Gerontologia Biomédica. Pontificia Universidade Católica Do Rio Grande do Sul.

\section{Vanessa Sgnaolin}

Farmacêutica. Doutora em Gerontologia Biomédica. Pontifícia Universidade Católica Do Rio Grande do Sul.

\section{Raquel Milani El Kik}

Nutricionista. Doutora em Gerontologia Biomédica. Pontifícia Universidade Católica do Rio Grande do Sul.

\section{Fernanda Bueno Morrone}

Farmacêutica. Doutorado em Ciências Biológicas Bioquímica. Pontificia Universidade Católica do Rio Grande do Sul.

\section{Irenio Gomes}

Médico. Doutor em Gerontologia Biomédica. Pontifícia Universidade Católica do Rio Grande do Sul.

\section{Endereço para correspondência}

Vanessa Sgnaolin

Pontificia Universidade Católica do Rio Grande do Sul

Av. Ipiranga, 6681, Prédio $81,7^{\circ}$ andar, sala 703

CEP 90619-900

Porto Alegre, RS, Brasil 